\title{
3D GRB JETS DRILLING THROUGH THE PROGENITOR
}

\author{
D. López-Cámara ${ }^{1}$
}

\begin{abstract}
We present, for the first time, three dimensional (3D) adaptive mesh refinement simulations of a GRB jet crossing a pre-SN progenitor (with resolutions comparable to the resolutions of 2D simulations) (Lopez-Camara et al. 2012). The morphology, Lorentz factor, and symmetries, will be discussed in this presentation. Basically the evolution is divided into three main phases: the jet moving inside the progenitor; the jet just about break out of the progenitor; and the phase where the jet has broken out of the star and is now moving through the circumstellar medium. The resolution and 3D effects are also discussed. Still, as with all numerical work, the current investigation lacks in several aspects and needs improvement as well as further investigations.
\end{abstract}

\section{Introduction}

In most cases, the study of the jet-star interaction has been performed numerically, with analytic models used only for guidance (Bromberg et al. 2011; Matzner 2003; Morsony et al. 2007). Even so, studying the propagation of a relativistic outflow that is continuously shocked by a much denser environment is not trivial since the length-scale of features in the relativistic material is typically $\sim R / \Gamma$ and therefore a large dynamical range is involved. When possible, adaptive mesh refinement codes have been adopted (Lazzati et al. 2009, 2010, 2011a; Morsony et al. 2007, 2010; Nagakura et al. 2011), and the simulations have been limited to two dimensions (Aloy et al. 2000; Lazzati et al. 2009, 2010, 2011a; MacFadyen \& Woosley 1999; MacFadyen et al. 2001; Mizuta \& Aloy 2009; Mizuta et al. 2006; Morsony et al. 2007, 2010; Nagakura et al. 2011; Zhang et al. 2003). These studies have shown that even though the jet material is relativistic, the jet-head propagates sub-relativistically inside the star, thereby allowing causal contact between the bow shock at the head of the jet and the star. The shocked star material therefore

\footnotetext{
1 Department of Physics, NC State University, 2401 Stinson Drive, Raleigh, NC 27695-8202, USA
} 
drains at the sides of the jet producing a hot cocoon instead of being entrained in the jet.

Two dimensional simulations can provide important answers to the outstanding questions listed above. However, they are plagued by artifacts due to the presence of a symmetry axis in the center of the jet. While 3D simulations of GRB jets have been attempted in the past (Zhang et al. 2004), they were performed with a fixed grid code, casting doubt on the capability to resolve the required small scales. A 3D test case with AMR was presented by Wang et al. (2008), but since their jet-progenitor evolution did not converge as a function of the numerical resolution (unlike our study), not much could be inferred from their study. Thus, in this paper we present, for the first time, 3D adaptive mesh refinement (AMR) simulations of GRB jets crossing a pre-SN progenitor and then flowing through the circumstellar medium (CSM).

\section{Initial setup}

We consider the one-dimensional pre-supernova 16TI model from Woosley \& Heger (2006) as our initial stellar configuration. The pre-SN progenitor was immersed in a CSM with constant density $\left(\rho_{C S M}=10^{-10} \mathrm{~g} \mathrm{~cm}^{-3}\right)$. A relativistic jet (with half-opening angle of $10^{\circ}$, constant luminosity of $5.33 \times 10^{50} \mathrm{erg} \mathrm{s}^{-1}$, and an initial Lorentz Factor of 5, see Lopez-Camara et al. 2012 for further details), was imposed at all times as a boundary condition. In order to follow the temporal evolution of our initial setup, we solved the 3D gas-dynamic equations using the FLASH code (3D relativistic version in cartesian coordinates) (Fryxell et al. 2000). The simulation domain covered the top half of the pre-SN progenitor star as well as the CSM it is immersed in. The boundaries were set at $\mathrm{y}_{\min }=10^{9} \mathrm{~cm}, \mathrm{y}_{\max }=$ $2.4 \times 10^{11} \mathrm{~cm}, \mathrm{x}_{\max }=-\mathrm{x}_{\min }=6 \times 10^{10} \mathrm{~cm}$, and $\mathrm{z}_{\max }=-\mathrm{z}_{\min }=6 \times 10^{10} \mathrm{~cm}$. Only the equatorial plane $\left(\mathrm{y}=\mathrm{y}_{\min }\right)$ was set with a reflective boundary condition, all other were set with transmission conditions. Even though it has been shown that close to the pre-SN's progenitor nucleus the neutrinos play an important role (López-Cámara et al. 2009), since the inner boundary was set so far away, $\mathrm{R}_{i} \sim 10^{9} \mathrm{~cm}$, equivalent to approximately $10^{4}$ gravitational radii, from the region where neutrinos dominate (and where the compacts object relativistic effects must be taken into consideration), the neutrino and general relativistic effects were safely ignored. Also, we ignored self gravity and the pre-SN's angular momentum. We justify this by pointing out that the dynamical timescale of the pre-SN is of order close to hours. Then, since the integration time in our numerical simulations is at most $20 \mathrm{~s}$, we were safe to assume that the pre-SN progenitor remained practically static at all times.

\section{Results and discussion}

In Figure 1 (left panel) we show the density stratification maps for $t=9.3 \mathrm{~s}$. The morphology of our system is divided into two main phases: the jet moving inside 

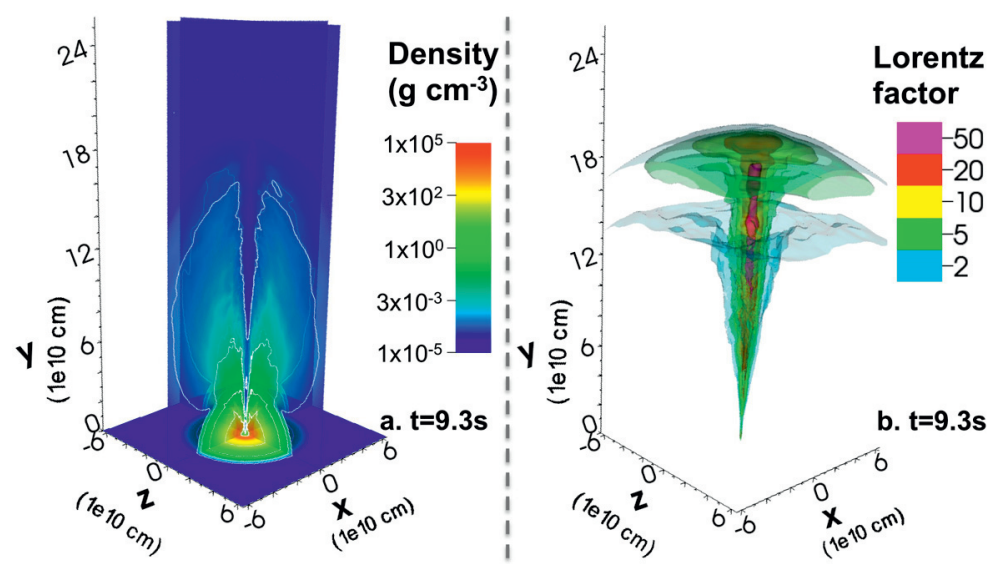

Fig. 1. Density stratification map $\left(\mathrm{g} \mathrm{cm}^{-3}\right)$ (left panel), and Lorentz factor isocontour map (right panel) at $\mathrm{t}=9.3 \mathrm{~s}$.

the progenitor; and when the jet has broken out of the progenitor (and is now interacting with the CSM).

The "break out time" from our simulations $\left(t_{b o}=4.2 \mathrm{~s}\right)$ implies that the average propagation velocity of the jet inside the star is $\sim 0.32 \mathrm{c}$. The jet, composed of low density material, has its initial opening angle reduced by relativistic hydrodynamic collimation effects. Before the break out time only a relativistic jet (with $\Gamma \sim 10$ ) is present. Once the jet breaks out of the stellar surface, the jet is accelerated and a cocoon forms. The high internal energy is able to accelerate material with Lorentz factors values of order $\Gamma \sim 10^{2}$ in some zones. To see the high Lorentz factor material in the jet, Figure 1 (right panel) we plot the $\Gamma$ isocontours for $\mathrm{t}=9.3 \mathrm{~s}$. By this time certain regions in the polar axis reach Lorentz factor values as high as $\Gamma=50$ (red isocontours).

In order to verify that the evolution of the jet from our results is not dependent on the numerical resolution, we ran a new model with the same setup and physics but with a maximum resolution two times finer than for the low resolution. In Figure 2 we show the density profiles for the three dimensional high resolution (3D $\mathrm{HR}$ ), and low resolution (3D LR) case, as well as for the 2D HR model. Among the differences associated with the resolution, are a higher level of turbulence and a slower advance of the jet head in the HR model. The reason the 3D HD jet to move slower (than the 3D LR) is due to the fact that the it has a wider jet. Since we are powering both jets equally, the narrow-LR jet will move faster. The reason for the $3 \mathrm{D}$ HR jet to show more less turbulence than its LR model is due to the fact that the 3D LR simulation has higher diffusion, and thus suppresses the small scale instabilities that are present in the HR model.

We also checked how the evolution of the jet through the stellar envelope and CSM differs in a 2D model. The basic morphology in the $2 \mathrm{D}$ case resembles that from the $3 \mathrm{D}$ model. In both cases we see a collimated jet that manages to drill 


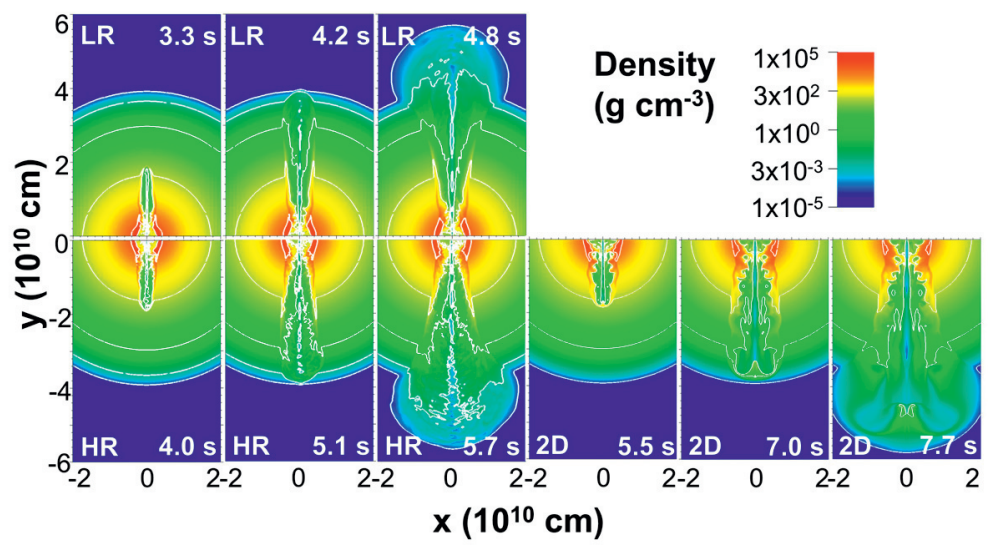

Fig. 2. Density stratification maps $\left(\mathrm{g} \mathrm{cm}^{-3}\right)$ for the $3 \mathrm{D}$ LR model (upper panels), for the $3 \mathrm{D}$ HR resolution model (lower left panels), and the 2D model (lower right panels).

through the stellar envelope. Apart from the cylindrical symmetry imposed in the $2 \mathrm{D}$ model, there are many subtle differences between the $2 \mathrm{D}$ and $3 \mathrm{D}$ results: The jet moves slower in the $2 \mathrm{D}$ model than in the $3 \mathrm{D}$ one, and the $2 \mathrm{D}$ jet presents less turbulent-like morphology. The reason for the $3 \mathrm{D}$ jet to move faster is due to the fact that the jet in $3 \mathrm{D}$ simulations is narrower and can wobble around the jet axis finding the path of least resistance to proceed. In Figure 3 we show the internal energy map in the XZ plane for the $3 \mathrm{D}$ scenario. The $\mathrm{XZ}$ planes shown for each timeframe correspond to the position where the internal energy's (U) centroid of the forward shock front was located at. It is clear how the centroid of the $U$ wobbles around the polar axis (contrary to the $2 \mathrm{D}$ case where it always moves through the polar axis).

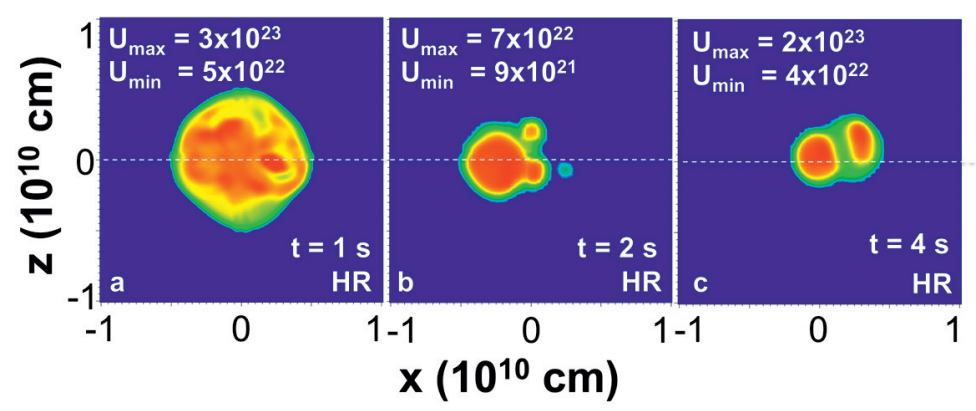

Fig. 3. Energy density $\left(\mathrm{erg} \mathrm{cm}^{3}\right) \mathrm{XZ}$ stratification maps for the centroid of the head front of the jet-cocoon structure for different times. 


\section{Conclusions}

We present, for the first time, 3D AMR simulations of GRB jets expanding inside a realistic pre-SN progenitor and then flowing through the CSM. Our numerical simulations, confirm that relativistic jets can propagate and break out of the progenitor star while remaining relativistic. The morphology is divided into two main phases: Pre- $t_{b o}$ phase in which the jet moves at mildly relativistic velocities $(\sim c / 2)$ inside the progenitor's stellar envelope; and the post- $t_{b o}$ phase in which the jet has broken out of the surface, it accelerates and reaches Lorentz factors of order $\Gamma \sim 50$. The resolution does not affect in great detail the flow and the morphology in each phase is well reproduced. Still, the amount of turbulence and variability observed in the simulations is higher for higher resolutions. Also, for finer numerical resolutions the jet moves slower. The propagation of the jet head inside the progenitor star is slightly faster in $3 \mathrm{D}$ simulations compared to $2 \mathrm{D}$ ones at the same resolution. This behavior is due to the fact that the jet in $3 \mathrm{D}$ simulations is narrower and can wobble around the jet axis finding the spot of least resistance to proceed.

\section{References}

Aloy, M.A., Müller, E., Ibáñez, J.M., Martí, J.M., \& MacFadyen, A., 2000, ApJ, 531, L119

Bromberg, O., Nakar, E., Piran, T., \& Sari, R., 2011, ApJ, 740, 100

Fryxell, B., Olson, K., Ricker, P., et al., 2000, ApJS, 131, 273

Lazzati, D., Morsony, B.J., \& Begelman, M.C., 2009, ApJ, 700, L47

Lazzati, D., Morsony, B.J., \& Begelman, M.C., 2010, ApJ, 717, 239

Lazzati, D., Morsony, B.J., \& Begelman, M.C., 2011, ApJ, 732, 34

López-Cámara, D., Lee, W.H., \& Ramirez-Ruiz, E., 2009, ApJ, 692, 804

López-Cámara, D., Morsony, B.J., Begelman, M.C., \& Lazzati, D., 2012 [arXiv: 1212.0539]

MacFadyen, A.I., \& Woosley, S.E., 1999, ApJ, 524, 262

MacFadyen, A.I., Woosley, S.E., \& Heger, A., 2001, ApJ, 550, 410

Matzner, C.D., 2003, MNRAS, 345, 575

Mizuta, A., \& Aloy, M.A., 2009, ApJ, 699, 1261

Mizuta, A., Yamasaki, T., Nagataki, S., \& Mineshige, S., 2006, ApJ, 651, 960

Morsony, B.J., Lazzati, D., \& Begelman, M.C., 2007, ApJ, 665, 569

Morsony, B.J., Lazzati, D., \& Begelman, M.C., 2010, ApJ, 723, 267

Nagakura, H., Ito, H., Kiuchi, K., \& Yamada, S., 2011, ApJ, 731, 80

Wang, P., Abel, T., \& Zhang, W., 2008, ApJS, 176, 467

Woosley, S.E., \& Heger, A., 2006, ApJ, 637, 914

Zhang, W., Woosley, S.E., \& Heger, A., 2004, ApJ, 608, 365

Zhang, W., Woosley, S.E., \& MacFadyen, A.I., 2003, ApJ, 586, 356 
